\title{
Survival Benefit of Adjuvant Radiation Therapy for Gastric Cancer following Gastrectomy and Extended Lymphadenectomy
}

\author{
R. A. Snyder, ${ }^{1}$ E. T. Castaldo, ${ }^{1}$ C. E. Bailey, ${ }^{1}$ S. E. Phillips, ${ }^{2}$ \\ A. B. Chakravarthy, ${ }^{3,4}$ and N. B. Merchant ${ }^{1,4,5}$ \\ ${ }^{1}$ Department of Surgery, Vanderbilt University Medical Center, Nashville, TN 37232, USA \\ ${ }^{2}$ Department of Biostatistics, Vanderbilt University Medical Center, Nashville, TN 37232, USA \\ ${ }^{3}$ Department of Radiation Oncology, Vanderbilt University Medical Center, Nashville, TN 37232, USA \\ ${ }^{4}$ Vanderbilt Ingram Cancer Center, Nashville, TN 37232-6860, USA \\ ${ }^{5}$ Division of Surgical Oncology, Vanderbilt University Medical Center, 597 Preston Research Building, Nashville, \\ TN 37232, USA
}

Correspondence should be addressed to N. B. Merchant, nipun.merchant@vanderbilt.edu

Received 30 January 2012; Accepted 6 May 2012

Academic Editor: Lapo Bencini

Copyright (C) 2012 R. A. Snyder et al. This is an open access article distributed under the Creative Commons Attribution License, which permits unrestricted use, distribution, and reproduction in any medium, provided the original work is properly cited.

\begin{abstract}
Purpose. Although randomized trials suggest a survival benefit of adjuvant chemotherapy and radiation therapy (XRT) for gastric adenocarcinoma, its use in patients who undergo an extended lymphadenectomy is less clear. The purpose of this study was to determine if a survival benefit exists in gastric cancer patients who receive adjuvant XRT following resection with extended lymphadenectomy. Methods. The SEER registry was queried for records of patients with resected gastric adenocarcinoma from 1988 to 2007. Multivariable Cox regression models were used to assess independent prognostic factors affecting overall survival (OS) and disease-specific survival (DSS). Results. Of 15,060 patients identified, 3,208 (21\%) received adjuvant XRT. Adjuvant XRT was independently associated with improved OS (HR 0.67, CI 0.64-0.71) and DSS (HR 0.69, CI 0.65-0.73) in stages IB through IV (M0). This OS and DSS benefit persisted regardless of the extent of lymphadenectomy. Furthermore, lymphadenectomy with $>25 \mathrm{LN}$ resected was associated with improved OS and DSS compared with $<15 \mathrm{LN}$ or $15-25 \mathrm{LN}$. Conclusion. This populationbased study shows a survival benefit of adjuvant XRT following gastrectomy that persists in patients who have an extended lymphadenectomy. Furthermore, removal of $>25$ LNs results in improved OS and DSS compared with patients who have fewer LNs resected.
\end{abstract}

\section{Introduction}

Gastric cancer is the fourth most common cancer and second leading cause of cancer-related deaths worldwide [1]. It has been estimated that there were 21,000 new cases of gastric cancer and 10,570 deaths from gastric cancer in the United States in 2010 [2]. Most patients in the U.S. present with locally advanced disease in which the tumor penetrates the muscularis propria and/or involves the perigastric lymph nodes at the time of diagnosis [3]. Surgical resection remains the only curative option for gastric adenocarcinoma. However, locoregional and systemic recurrence rates remain high, and ten-year overall survival (OS) rates after resection with curative intent range from $3-42 \%$ for advanced disease [4-6]. Given the high rates of recurrence after resection, the additional use of adjuvant chemotherapy and radiation therapy (XRT) has been investigated.

In 2001, the US Intergroup study (INT-0116) demonstrated an improvement in OS for patients with stage IB through IV (M0) gastric cancer who underwent resection followed by adjuvant 5-FU-based chemotherapy and XRT compared with patients who underwent surgical resection alone [7]. Based on these results, this adjuvant therapy regimen became the standard of care for resectable gastric cancer in the U.S. A retrospective observational study using the Surveillance, Epidemiology, and End Results (SEER)Medicare database reproduced these findings, demonstrating an improvement in OS for patients with gastric cancer who received adjuvant chemoradiation therapy [8]. 
In the INT-0116 trial, a D2 lymph node dissection (resection of regional lymphatics and perigastric lymph nodes (LNs), as well as LNs along the named vessels of the celiac axis) was recommended in all patients. However, only $10 \%$ of patients actually received this level of nodal clearance. In fact, $54 \%$ of patients underwent a D0 resection (gastrectomy with incomplete resection of the N1 nodes) [7]. This calls into question whether adjuvant chemoradiation therapy was associated with improved disease-free survival (DFS) and OS in this trial by decreasing locoregional recurrence in patients who underwent an inadequate lymph node dissection (LND). In a post hoc analysis of INT-0116, there was no significant evidence that chemoradiation failed to work in the D2 subgroup; however, with only 54 patients in this group, the authors acknowledge that the power of this analysis was very low [9].

The primary aim of this study, therefore, was to evaluate whether the survival benefit of adjuvant chemoradiation therapy persists in patients undergoing gastrectomy and extended lymphadenectomy by using a large, national database that could provide significant statistical power to detect a survival difference.

\section{Materials and Methods}

A data set consisting of patients with gastric adenocarcinoma was created through a query of the SEER database. SEER is an authoritative source for cancer incidence, survival, and prevalence encompassing approximately $28 \%$ of the United States population [10]. The SEER program collects demographic information (e.g., age, gender, and race) and clinical information (e.g., primary tumor site, tumor histology, tumor grade, stage, treatment, and survival) from 17 cancer registries across the United States. Stage information from the SEER database was converted to the American Joint Committee on Cancer 6th edition (AJCC) tumor node metastasis (TNM) criteria.

All patient records in the SEER registry from 1988 to 2007 with surgically resected gastric adenocarcinoma were queried ( $n=78,511)$. Patients in whom gastric adenocarcinoma was not the primary malignancy or patients who lacked a histologically confirmed diagnosis of gastric adenocarcinoma were excluded from analysis. Patients with gastric lymphoma, gastrointestinal stromal tumors, or other gastric malignancies were excluded. In addition, those patients who had incomplete clinicopathologic information, metastatic disease, or who had undergone preoperative or intraoperative XRT were excluded.

Statistical analysis was performed using a Cox regression model with gender, race (categorized as white, black, and other), age, number of LNs resected, AJCC stage of disease, and radiation status as covariates. The primary and secondary outcome measures were OS and DSS, respectively. Kaplan-Meier methods and the log-rank test were also used to compare survival between patients who did or did not receive adjuvant XRT according to stage and extent of LND. For purposes of statistical analysis, the number of LNs resected was subdivided into groups of $<15$ LNs, 15-25 LNs, and $>25$ LNs. Age was dichotomized to $\leq 60$ years and
$>60$ years of age. All $P$ values were two-tailed tests, with alpha of 0.05 .

The Vanderbilt Institutional Review Board (IRB) was contacted regarding this study; however, because the data from SEER is de-identified prior to release to our institution, no formal IRB approval was required.

\section{Results}

A total of 15,060 patients met the inclusion criteria. Of these, $3,208(21 \%)$ received adjuvant XRT after gastric resection and 11,852 (79\%) underwent gastric resection alone. Patient characteristics are shown in Table 1. Several statistical differences between patient populations were identified. Patients of younger age $(\leq 60)$, male gender, and higher stage tumors were more likely to receive adjuvant XRT $(P<0.001)$. Patients who had $\geq 15$ LNs removed were also more likely to receive adjuvant XRT $(P<0.001)$.

Kaplan Meier survival analysis demonstrated a significant 5 year OS benefit of adjuvant XRT when compared with surgery alone for all patients with AJCC stage IB $(P=$ $0.002)$ and higher $(P<0.001)$. Median OS among patients with stage IB, II, IIIA, and IIIB gastric cancer who received adjuvant XRT was 65, 34, 23, and 19 months, respectively, compared with 54,21,14, and 11 months, respectively, for patients who underwent surgery alone. These results are summarized in Table 2. Five year DSS was also improved for patients with stage II and higher who received adjuvant XRT $(P<0.001)$. Median DSS for patients who received adjuvant XRT with stages II, IIIA, and IIIB was 41, 24, and 20 months, respectively, compared with 26, 17, and 12 months for patients who had surgery alone.

An extended LND was also associated with improved OS by Kaplan Meier analysis. Median survival was 34 months for patients who had $>25$ LNs resected, 27 months if 15-25 LNs were resected, and 25 months if $<15$ LNs resected $(P<$ $0.001)$. Five-year OS was 38\% when $>25$ LNs resected, $33 \%$ with 15-25 LNs resected, and 31\% if $<15$ LNs were resected (see Figure 1). Median DSS was also improved with a more extended LND: 45 months for patients with resection of $>25$ LN, 34 months for 15-25 LNs, and 32 months for $<15$ LNs $(P<0.001)$ (Figure 2).

By Cox multivariate regression analysis, adjuvant XRT was independently associated with improved OS (HR 0.67, CI 0.64-0.71) and DSS (HR 0.69, CI 0.65-0.73) in patients with stages IB through IV (M0) undergoing resection for gastric cancer (see Tables 3 and 4). Notably, when extent of lymphadenectomy was included as a covariate in this analysis with patients stratified into groups according to the number of LNs resected ( $<15 \mathrm{LN}, 15-25 \mathrm{LN}$, or $>25 \mathrm{LN}$ ), the demonstrated OS and DSS benefit of adjuvant XRT persisted regardless of the extent of lymphadenectomy. In this model, a more extended LND ( $>25 \mathrm{LN}$ ) was also independently associated with improved OS and DSS when compared with a less extensive LND (<15 LN-OS HR 0.65, CI 0.60-0.69; DSS HR 0.62, CI $0.57-0.67$ or 15-25 LN-OS HR 0.84, CI $0.78-$ 0.91; DSS HR 0.81, CI 0.75-0.88).

Additional variables that were independently associated with improved OS and DSS included female gender 
TABle 1: Patient characteristics.

\begin{tabular}{|c|c|c|c|c|c|}
\hline & \multicolumn{2}{|c|}{ No XRT } & \multicolumn{2}{|c|}{ XRT } & \multirow{2}{*}{$P$ value } \\
\hline & $N$ & $\%$ & $N$ & $\%$ & \\
\hline$N$ & 11852 & 100 & 3208 & 100 & \\
\hline \multicolumn{6}{|l|}{ Age } \\
\hline$\leq 60$ & 2344 & 20 & 1286 & 40 & \multirow{2}{*}{$<0.001$} \\
\hline$>60$ & 9508 & 80 & 1922 & 60 & \\
\hline \multicolumn{6}{|l|}{ Sex } \\
\hline Male & 7452 & 63 & 2234 & 70 & \multirow{2}{*}{$<0.001$} \\
\hline Female & 4400 & 37 & 974 & 30 & \\
\hline \multicolumn{6}{|l|}{ Race } \\
\hline White & 7926 & 67 & 2187 & 68 & \multirow{3}{*}{0.284} \\
\hline Black & 1364 & 12 & 368 & 11 & \\
\hline Other & 2562 & 22 & 653 & 20 & \\
\hline \multicolumn{6}{|l|}{ LN dissection } \\
\hline$<15$ nodes & 7731 & 67 & 1823 & 58 & \multirow{3}{*}{$<0.001$} \\
\hline $15-25$ nodes & 2633 & 23 & 912 & 29 & \\
\hline$>25$ nodes & 1141 & 10 & 424 & 13 & \\
\hline \multicolumn{6}{|l|}{ Grade } \\
\hline I & 820 & 7 & 86 & 3 & \multirow{4}{*}{$<0.001$} \\
\hline II & 4213 & 36 & 919 & 29 & \\
\hline III & 6583 & 56 & 2129 & 66 & \\
\hline IV & 236 & 2 & 74 & 2 & \\
\hline \multicolumn{6}{|l|}{ Stage } \\
\hline IA & 2207 & 19 & 31 & 1 & \multirow{6}{*}{$<0.001$} \\
\hline IB & 2717 & 23 & 503 & 16 & \\
\hline II & 3146 & 27 & 1183 & 37 & \\
\hline IIIA & 2041 & 17 & 817 & 25 & \\
\hline IIIB & 395 & 3 & 212 & 7 & \\
\hline IV (M0) & 1346 & 11 & 462 & 14 & \\
\hline
\end{tabular}

TABLE 2: Overall survival by stage.

\begin{tabular}{|c|c|c|c|c|c|}
\hline \multirow{2}{*}{ Stage } & \multicolumn{2}{|c|}{ No XRT } & \multicolumn{2}{|r|}{ XRT } & \multirow[t]{2}{*}{$P$ value } \\
\hline & $N$ & Median survival (mo) & $N$ & Median survival (mo) & \\
\hline All & 11852 & 25 & 3208 & 28 & $<0.001$ \\
\hline IA & 2207 & 114 & 31 & 86 & 0.969 \\
\hline IB & 2717 & 54 & 503 & 65 & 0.002 \\
\hline II & 3146 & 21 & 1183 & 34 & $<0.001$ \\
\hline IIIA & 2041 & 14 & 817 & 23 & $<0.001$ \\
\hline IIIB & 395 & 11 & 212 & 19 & $<0.001$ \\
\hline IV (M0) & 1346 & 9 & 462 & 17 & $<0.001$ \\
\hline
\end{tabular}

$(P<0.001)$, race other than black $(P<0.001)$, younger age $(\leq 60)(P<0.001)$, and lower tumor stage $(P<0.001)$ as shown in Tables 3 and 4.

\section{Discussion}

The primary aim of our study was to determine whether adjuvant radiation therapy provides a survival benefit specifically to patients who have undergone an extended lymphadenectomy. To answer this question, we used data from SEER, a large, national database statistically powered to detect differences in OS and DSS. Using a multivariate analysis of over 15,000 patients, we demonstrate that an OS and DSS advantage persists in patients receiving adjuvant XRT even in the subgroup who have undergone an extended LND ( $>25 \mathrm{LN})$. The strength of this analysis lies in the inclusion of a large number of patients who underwent extended lymphadenectomy $(N=1,565)$. Although a subgroup analysis of patients who underwent D2 resection in INT-0116 was performed, the analysis was underpowered due to the small number of patients studied $(N=54)$.

The extent of lymphadenectomy accompanying gastrectomy remains controversial. An extended LND is thought to decrease locoregional recurrence and provide more accurate 


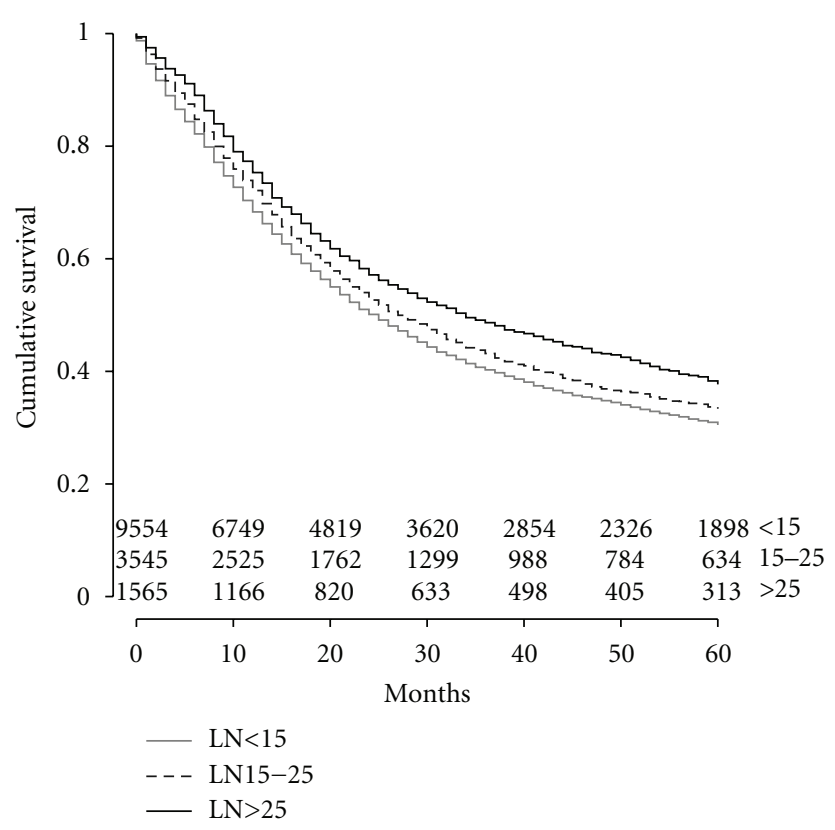

Figure 1: Kaplan Meier curve of overall survival by lymph node resection. Lymph node (LN).

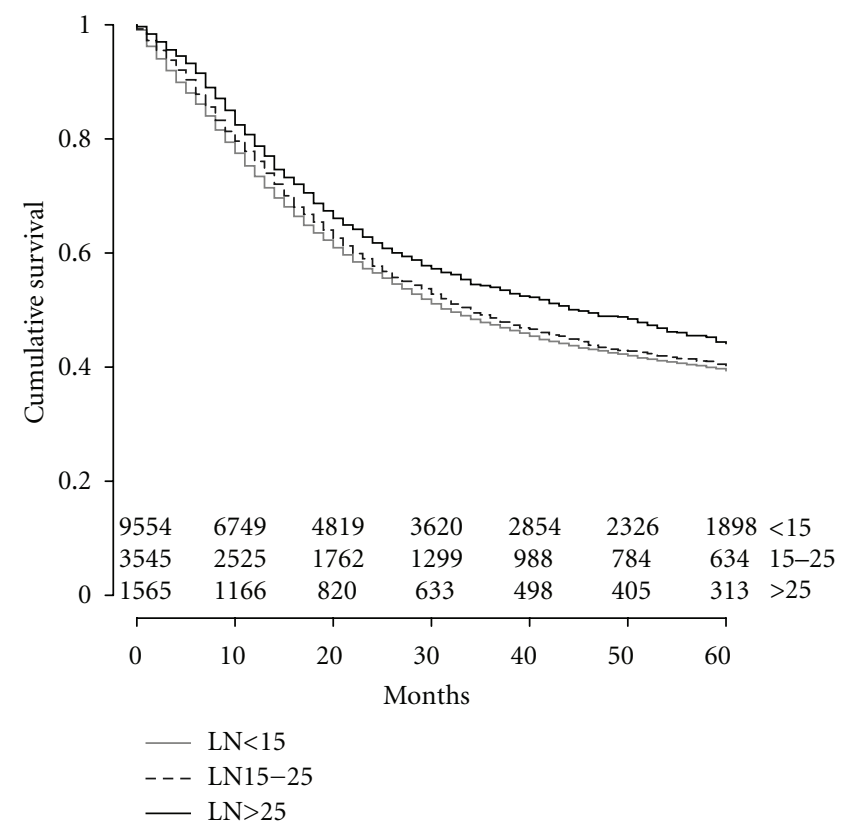

Figure 2: Kaplan Meier curve of disease specific survival by lymph node resection. Lymph node (LN).

staging information. Although Japanese series have consistently shown a significant survival benefit with D2 LND, two large European randomized controlled trials, the Dutch Gastric Cancer Trial (DGCT) and the UK Medical Research Council (MRC) trial, failed to demonstrate this benefit [1117]. In both of these trials, the morbidity and mortality of patients who underwent a D2 LND was significantly higher than patients who underwent a limited (D1) LND $[17,18]$.
However, a subset analysis of DGCT found a survival benefit of D2 LND for stages II and IIIA, a finding also demonstrated by a large, prospective German trial $[16,19]$. Furthermore, a subset analysis of the MRC trial demonstrated superior survival for patients who underwent a D2 LND without splenectomy or pancreatectomy, indicating that the high-operative mortality may have masked the survival benefit of a D2 LND [17]. Retrospective reviews in the USA using SEER and the National Cancer Database have similarly demonstrated an improved OS after extended lymphadenectomy for patients with stages II-IV gastric cancer $[20,21]$.

The long-term results of DGCT have been recently released and indicate that $\mathrm{D} 2$ lymphadenectomy is associated with reduced locoregional recurrence rates and improved DSS, and the authors therefore recommend a modified spleen-preserving D2 lymphadenectomy [6]. In addition, the National Comprehensive Cancer Network (NCCN) has recently modified their recommendations to include a D2 lymphadenectomy for patients with resectable gastric cancer [22].

The D level of a lymphadenectomy is defined by the Japanese Research Society for the Study of Gastric Cancer (JRSGC) based on the location of the primary tumor in the stomach and the level of regional lymph node station involvement [9]. However, the number of lymph nodes removed can also be used to approximate the extent of LND. According to the Japanese literature, a radical lymphadenectomy corresponding to a D2 resection consists of removal of 26 or more nodes [23]. In a Korean study of 990 patients who underwent curative resection with negative margins and D2 lymphadenectomy, $87 \%$ of patients had $>25$ nodes resected [24]. In the Dutch randomized trial comparing D1 and D2 lymphadenectomy, the median number of nodes removed was 17 and 30, respectively [16]. This suggests that a D2 lymphadenectomy correlates with resection of approximately 25 or more LNs. Thus, when the anatomic nodal location is unknown, the level of LND (D1 or D2) can be approximated using the total number of resected LNs. It was necessary to use this definition in our analysis, as SEER data consists of the number of $\mathrm{LN}$ removed but does not include information about anatomic nodal stations.

In this study, we also found a survival benefit of adjuvant XRT after surgical resection in patients with stage IB through stage IV (M0) gastric cancer. Median survival improvement ranged from 8 months to 13 months depending on stage. These findings correspond to and confirm the results of the INT-0116 trial, which showed a 9 month median survival advantage with the use of adjuvant XRT and 5-FU [7].

Additionally, our study shows that a more extended LND in-and-of-itself results in improved OS and DSS with the survival benefit increasing as the extent of lymphadenectomy increases. These findings suggest that patients with gastric cancer should undergo a margin negative resection with an extended ( $>25$ nodes or D2) lymphadenectomy and confirms that adjuvant radiation therapy is an important component of their treatment.

There are several limitations to this study. First, data on the use of chemotherapy is unavailable for analysis using 
TABLE 3: Cox multivariate regression analysis for overall survival.

\begin{tabular}{|c|c|c|c|}
\hline Variable & HR & $95 \% \mathrm{CI}$ & $P$ value \\
\hline No XRT & 1.00 & Reference & \multirow{2}{*}{$<0.001$} \\
\hline Adjuvant XRT & 0.67 & $0.64-0.71$ & \\
\hline \multicolumn{4}{|l|}{ Age } \\
\hline$\leq 60$ & 1.00 & Reference & \multirow{2}{*}{$<0.001$} \\
\hline$>60$ & 1.49 & $1.42-1.57$ & \\
\hline \multicolumn{4}{|l|}{ Gender } \\
\hline Male & 1.00 & Reference & \multirow{2}{*}{$<0.001$} \\
\hline Female & 0.88 & $0.84-0.91$ & \\
\hline \multicolumn{4}{|l|}{ Race } \\
\hline White & 1.00 & Reference & \\
\hline Black & 1.06 & $0.99-1.13$ & 0.075 \\
\hline Other & 0.77 & $0.73-0.81$ & $<0.001$ \\
\hline \multicolumn{4}{|l|}{ Lymph nodes } \\
\hline $\mathrm{LN}<15:>25$ & 0.65 & $0.60-0.69$ & $<0.001$ \\
\hline LN $15-26:>25$ & 0.84 & $0.78-0.91$ & $<0.001$ \\
\hline \multicolumn{4}{|l|}{ Stage } \\
\hline IA & 1.00 & Reference & \\
\hline IB & 1.689 & $1.55-1.84$ & 0.004 \\
\hline II & 3.08 & $2.84-3.35$ & $<0.001$ \\
\hline IIIA & 4.44 & $4.08-4.83$ & $<0.001$ \\
\hline IIIB & 6.02 & $5.34-6.78$ & 0.003 \\
\hline IV (M0) & 7.14 & $6.52-7.82$ & $<0.001$ \\
\hline
\end{tabular}

TABLE 4: Cox multivariate regression analysis for disease-specific survival.

\begin{tabular}{|c|c|c|c|}
\hline Variable & HR & $95 \% \mathrm{CI}$ & $P$ value \\
\hline No XRT & 1.00 & Reference & \multirow{2}{*}{$<0.001$} \\
\hline Adjuvant XRT & 0.69 & $0.65-0.73$ & \\
\hline \multicolumn{4}{|l|}{ Age } \\
\hline$\leq 60$ & 1.00 & Reference & \multirow{2}{*}{$<0.001$} \\
\hline$>60$ & 1.26 & $1.19-1.33$ & \\
\hline \multicolumn{4}{|l|}{ Gender } \\
\hline Male & 1.00 & Reference & \multirow{2}{*}{$<0.001$} \\
\hline Female & 0.88 & $0.84-0.93$ & \\
\hline \multicolumn{4}{|l|}{ Race } \\
\hline White & 1.00 & Reference & \\
\hline Black & 1.04 & $0.97-1.11$ & 0.307 \\
\hline Other & 0.75 & $0.71-0.80$ & $<0.001$ \\
\hline \multicolumn{4}{|l|}{ Lymph nodes } \\
\hline $\mathrm{LN}<15:>25$ & 0.62 & $0.57-0.67$ & $<0.001$ \\
\hline LN 15-26: >25 & 0.81 & $0.75-0.88$ & $<0.001$ \\
\hline \multicolumn{4}{|l|}{ Stage } \\
\hline IA & 1.00 & Reference & \\
\hline IB & 2.47 & $2.18-2.80$ & $<0.001$ \\
\hline II & 5.38 & $4.78-6.05$ & $<0.001$ \\
\hline IIIA & 8.32 & $7.38-9.39$ & $<0.001$ \\
\hline IIIB & 11.31 & $9.74-13.12$ & $<0.001$ \\
\hline IV (M0) & 13.78 & $12.16-15.61$ & $<0.001$ \\
\hline
\end{tabular}


the SEER database. Although adjuvant 5-FU chemotherapy and XRT was the standard of care during the study period, it is certainly possible that patients may have received an alternative adjuvant regimen. Some patients may have undergone surgery/5-FU only or surgery/XRT only. As these are not standard adjuvant therapy regimens, it is likely that the number of patients falling into these categories is low. In one study using SEER-Medicare data in which chemotherapy use is known, fewer than $15 \%$ of the 2,333 patients received surgery and either chemotherapy or radiation alone [8]. Further, the MAGIC trial, which demonstrated a benefit of perioperative chemotherapy was published in 2006, so it is unlikely that a significant proportion of patients received chemotherapy alone outside of a clinical trial during the dates of this study [25].

SEER data also does not include information about local or distant recurrence; therefore, disease-free survival (DFS) cannot be determined. Additionally, there is no information about margin status after resection or about the dose or details of the radiation administered.

\section{Conclusions}

In summary, this study supports the use of adjuvant XRT in the treatment of gastric adenocarcinoma, as it appears to improve OS and DSS in patients with stage IB-IV (M0) gastric cancer. More importantly, our findings demonstrate that the survival benefit of XRT persists regardless of the extent of lymphadenectomy. This suggests that the benefit of XRT is not simply a mechanism to compensate for inadequate surgical clearance of disease, but is in itself critical for achieving locoregional control. Future prospective studies should include the use of adjuvant XRT and extended LND as independent variables to validate these findings.

\section{References}

[1] K. D. Crew and A. I. Neugut, "Epidemiology of gastric cancer," World Journal of Gastroenterology, vol. 12, no. 3, pp. 354-362, 2006.

[2] S. F. K. C. Altekruse, M. Krapcho, N. Neyman et al., SEER Cancer Statistics Review, 1975-2007, National Cancer Institute, Bethesda, Md, USA, 2010.

[3] W. B. Al-Refaie, J. F. Tseng, G. Gay et al., "The impact of ethnicity on the presentation and prognosis of patients with gastric adenocarcinoma: results from the national cancer data base," Cancer, vol. 113, no. 3, pp. 461-469, 2008.

[4] S. A. Hundahl, J. L. Phillips, and M. R. Menck, "The National Cancer Data Base Report on poor survival of U.S. gastric carcinoma patients treated with gastrectomy. Fifth Edition American Joint Committee on Cancer staging, proximal disease, and the " different disease" hypothesis," Cancer, vol. 88, no. 4, pp. 921-932, 2000.

[5] H. J. Wanebo, B. J. Kennedy, J. Chmiel, G. Steele, D. Winchester, and R. Osteen, "Cancer of the stomach: a patient care study by the American College of Surgeons," Annals of Surgery, vol. 218, no. 5, pp. 583-592, 1993.

[6] I. Songun, H. Putter, E. M. Kranenbarg, M. Sasako, and C. J. H. van de Velde, "Surgical treatment of gastric cancer: 15-year follow-up results of the randomised nationwide Dutch D1D2 trial," The Lancet Oncology, vol. 11, no. 5, pp. 439-449, 2010.
[7] J. S. Macdonald, S. R. Smalley, J. Benedetti et al., "Chemoradiotherapy after surgery compared with surgery alone for adenocarcinoma of the stomach or gastroesophageal junction," The New England Journal of Medicine, vol. 345, no. 10, pp. 725730, 2001.

[8] J. Strauss, D. L. Hershman, D. Buono et al., "Use of adjuvant 5-fluorouracil and radiation therapy after gastric cancer resection among the elderly and impact on survival," International Journal of Radiation Oncology Biology Physics, vol. 76, no. 5, pp. 1404-1412, 2010.

[9] S. A. Hundahl, J. S. Macdonald, J. Benedetti, and T. Fitzsimmons, "Surgical treatment variation in a prospective, randomized trial of chemoradiotherapy in gastric cancer: the effect of undertreatment," Annals of Surgical Oncology, vol. 9, no. 3, pp. 278-286, 2002.

[10] M. J. Hayat, N. Howlader, M. E. Reichman, and B. K. Edwards, "Cancer statistics, trends, and multiple primary cancer analyses from the surveillance, epidemiology, and end results (SEER) program," Oncologist, vol. 12, no. 1, pp. 20-37, 2007.

[11] K. Maruyama, P. Gunven, K. Okabayashi, M. Sasako, and T. Kinoshita, "Lymph node metastases of gastric cancer. General pattern in 1931 patients," Annals of Surgery, vol. 210, no. 5, pp. 596-602, 1989.

[12] K. Maruyama, K. Okabayashi, and T. Kinoshita, "Progress in gastric cancer surgery in Japan and its limits of radicality," World Journal of Surgery, vol. 11, no. 4, pp. 418-425, 1987.

[13] Y. Kodera, Y. Yamamura, A. Torii et al., "Postoperative staging of gastric carcinoma: a comparison between the UICC stage classification and the 12th edition of the Japanese general rules for gastric cancer study," Scandinavian Journal of Gastroenterology, vol. 31, no. 5, pp. 476-480, 1996.

[14] M. Sasako, P. McCullouch, T. Kinoshita, and K. Maruyama, "New method to evaluate the therapeutic value of lymph node dissection for gastric cancer," British Journal of Surgery, vol. 82, no. 3, pp. 346-351, 1995.

[15] Y. Kodama, K. Sugimachi, and K. Soejima, "Evaluation of extensive lymph node dissection for carcinoma of the stomach," World Journal of Surgery, vol. 5, no. 2, pp. 241-248, 1981.

[16] J. J. Bonenkamp, J. Hermans, M. Sasako, and C. J. H. Van De Velde, "Extended lymph-node dissection for gastric cancer," The New England Journal of Medicine, vol. 340, no. 12, pp. 908914, 1999.

[17] A. Cuschieri, S. Weeden, J. Fielding et al., "Patient survival after $D_{1}$ and $D_{2}$ resections for gastric cancer: long-term results of the MRC randomized surgical trial," British Journal of Cancer, vol. 79, no. 9-10, pp. 1522-1530, 1999.

[18] H. H. Hartgritik, C. J. van de Velde, H. Putter et al., "Extended lymph node dissection for gastric cancer: who may benefit? Final results of the randomized Dutch gastric cancer group trial," Journal of Clinical Oncology, vol. 22, no. 11, pp. 20692077, 2004.

[19] J. R. Siewert, K. Bottcher, J. D. Roder, R. Busch, P. Hermanek, and H. J. Meyer, "Prognostic relevance of systematic lymph node dissection in gastric carcinoma," British Journal of Surgery, vol. 80, no. 8, pp. 1015-1018, 1993.

[20] R. E. Schwarz and D. D. Smith, "Clinical impact of lymphadenectomy extent in resectable gastric cancer of advanced stage," Annals of Surgical Oncology, vol. 14, no. 2, pp. 317-328, 2007.

[21] D. D. Smith, R. R. Schwarz, and R. E. Schwarz, "Impact of total lymph node count on staging and survival after gastrectomy for gastric cancer: data from a large US-population database," 
Journal of Clinical Oncology, vol. 23, no. 28, pp. 7114-7124, 2005.

[22] NCCN, NCCN Clinical Practice Guidelines in Oncology: Gastric Cancer, National Comprehensive Cancer Network, version 2.2011 edition, 2011.

[23] T. Kajitani, "The general rules for the gastric cancer study in surgery and pathology_part I. Clinical classification," Japanese Journal of Surgery, vol. 11, no. 2, pp. 127-139, 1981.

[24] S. Kim, D. H. Lim, J. Lee et al., "An observational study suggesting clinical benefit for adjuvant postoperative chemoradiation in a population of over 500 cases after gastric resection with D2 nodal dissection for adenocarcinoma of the stomach," International Journal of Radiation Oncology Biology Physics, vol. 63, no. 5, pp. 1279-1285, 2005.

[25] D. Cunningham, W. H. Allum, S. P. Stenning et al., "Perioperative chemotherapy versus surgery alone for resectable gastroesophageal cancer," The New England Journal of Medicine, vol. 355, no. 1, pp. 11-20, 2006. 


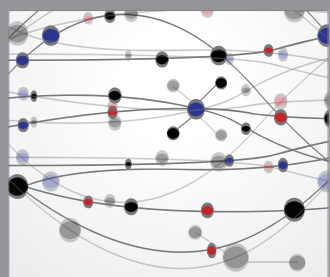

The Scientific World Journal
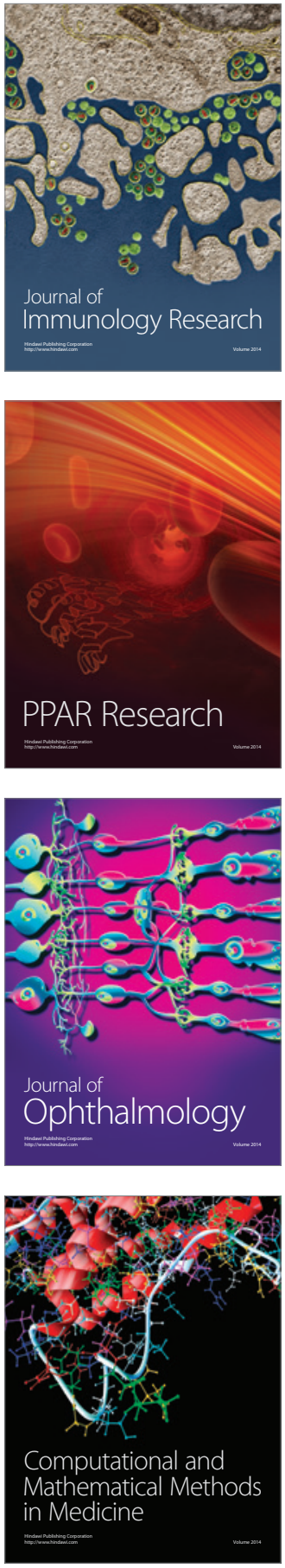

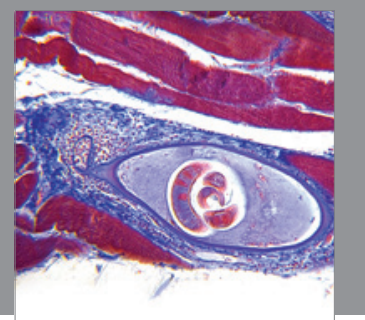

Gastroenterology

Research and Practice
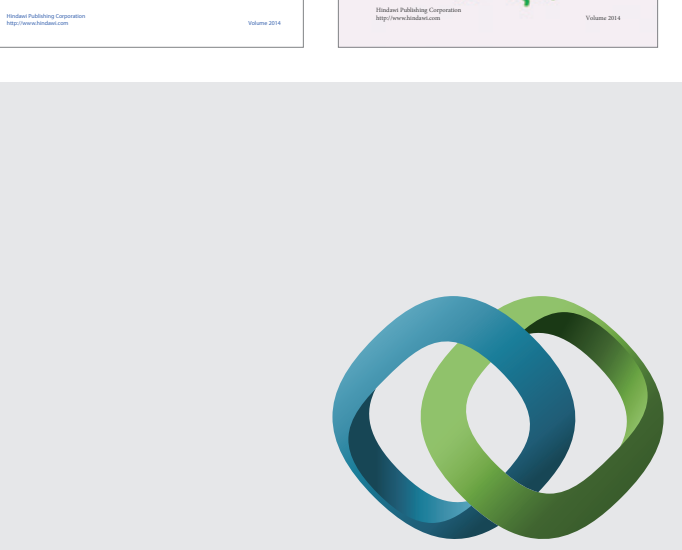

\section{Hindawi}

Submit your manuscripts at

http://www.hindawi.com
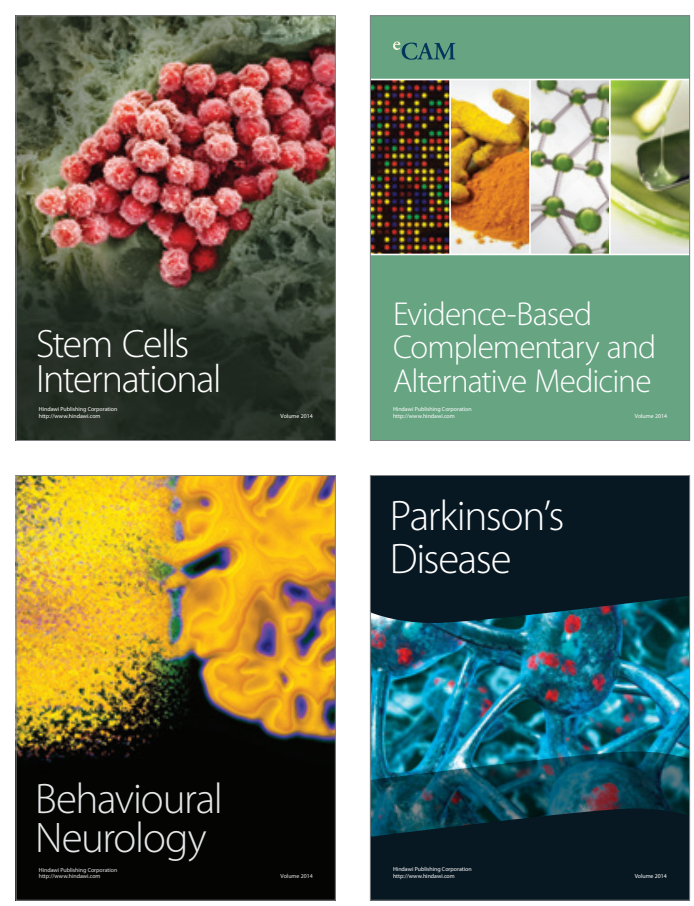

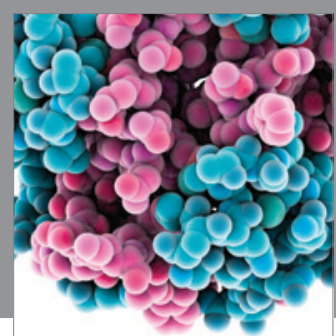

Journal of
Diabetes Research

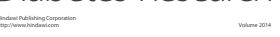

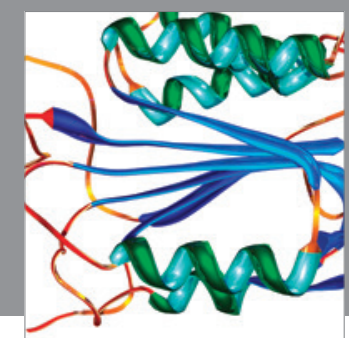

Disease Markers
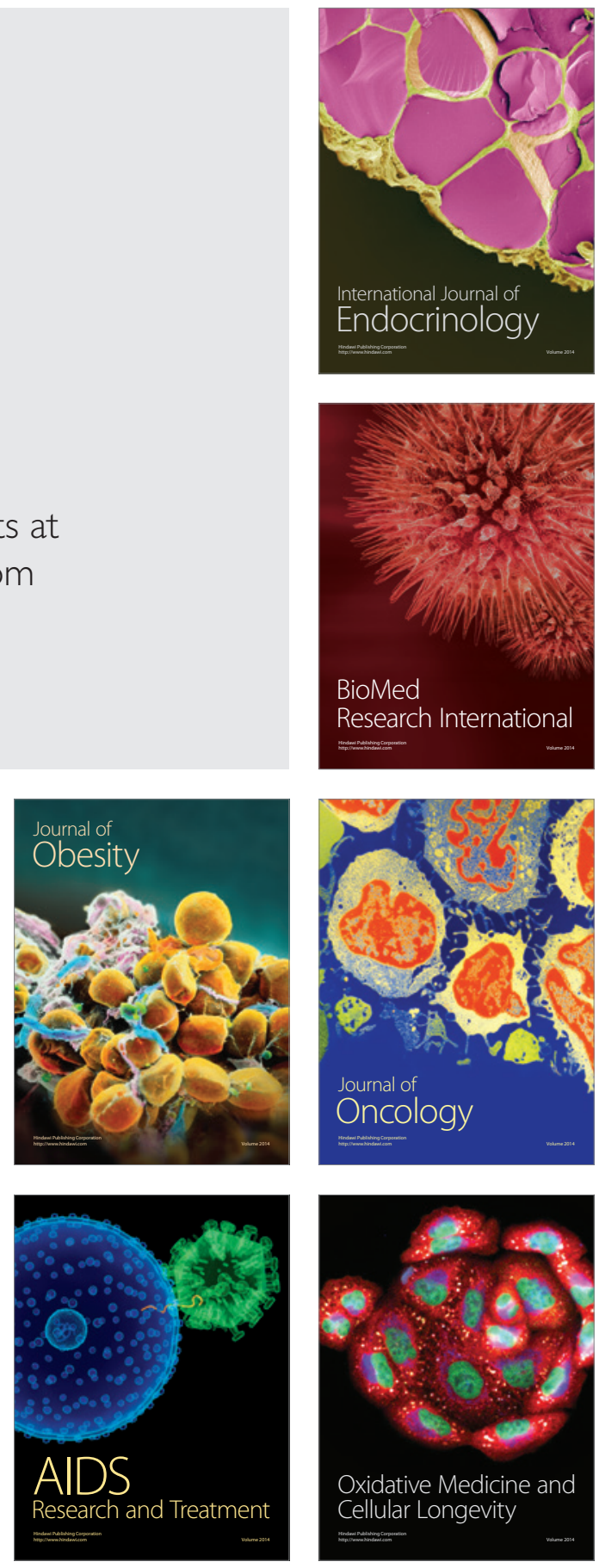